\title{
Case Management - Kooperation im Dienste des Patienten
}

\author{
Aus der Versichererperspektive brachte die Einführung der 5. IVG-Revision einige Ver- \\ besserungen. So konnte der IV-Neurentenzuwachs stabilisiert werden, und die von \\ Patienten wahrgenommenen «Subjektiv-Krankempfindungen» wurden als Renten- \\ grund ausgeschlossen. Die Erfahrungen an der Schadenfront zeigen jedoch deutlich, \\ dass die Schadensbearbeitungsmodalitäten für die Patienten viel härter geworden \\ sind. Ein Fallbeispiel.
}

\section{Ruedi Schläppi}

Eidg. dipl. Privat- und Sozialversicherungsexperte sowie institutionsunabhängiger Case Manager NDK-HSA
Interessenbindungen: Der Autor betreibt eine unabhängige Koordinationsstelle für Beratung, Koordination, Schadenbegleitung, Integration, Rehabilitation und Schulung im Privat- und Sozialversicherungsbereich. Das im Artikel vorgestellte Fallbeispiel stammt aus seiner Praxis.

\section{Korrespondenz:}

Ruedi Schläppi

Mühleried

CH-6213 Knutwil

Tel. 0419221246

Fax 0419221248

info@koordinationsstelle.ch
Begriffe wie «Case Management (CM)», «iiz.ch», «iizplus.ch», «FeFi» und andere Fachbezeichnungen (siehe Kasten «Begriffsklärungen» auf der nächsten Seite) beherrschen in zunehmendem Masse die Versicherungswelt. Es handelt sich dabei um Instrumente, die es ermöglichen sollen, in einem immer komplexer werdenden Gesundheitssystem das für eine Patientin oder einen Patienten angestrebte Optimum in möglichst effizienter und für alle Beteiligten zuträglicher Weise zu erreichen. digung in Aussicht. Diese Entwicklung belastete die Patientin zusehends psychisch, so dass sie sich ab Oktober 2008 aus eigenem Antrieb in psychotherapeutische Behandlung begab.

$\mathrm{Zu}$ Beginn der Arbeitsunfähigkeit gewährte der Krankentaggeldversicherer des Arbeitgebers vollen Lohnersatz. Die Patientin wurde im Frühjahr 2009 auf Veranlassung des Taggeldversicherers zu einer vertrauensärztlichen Untersuchung einbestellt. Der Vertrauensarzt kam - wie das bei solchen Konstellationen oft

\section{Das einfache Benennen oder Umschreiben eines Krankenbildes genügt im Rahmen der Umsetzungsmodalitäten der 5. IVG-Revision nicht mehr}

Allerdings wird nicht in jedem Fall ein Case Management (CM) etabliert. Wenn bei einer verunfallten oder erkrankten Person gewisse Voraussetzungen ungünstig zusammentreffen, besteht für sie sogar eher eine geringe Chance, durch ein CM betreut zu werden (vgl. Kasten). Zudem können Situationen entstehen, die auch für die in die Behandlung involvierten Hausund Fachärzte sehr herausfordernd sind und diesen die Entwicklung neuer Denkweisen und Strategien abverlangen. Wie dies gemeint ist, soll im Folgenden anhand eines konkreten Falls erläutert werden.

\section{Fallbeispiel}

Bei einer Patientin mit einem dorsoradialen Handgelenks-Ganglion links, das im Frühling 2006 operiert worden war, verlief der Heilungsprozess nicht erwartungsgemäss. Verschiedene Behandlungsversuche brachten keine Fortschritte, und die Gründe für die persistierenden Beschwerden konnten nicht klar diagnostiziert werden. Die Erkrankung führte ab November 2008 zur Arbeitsunfähigkeit. Da die Situation stagnierte, stellte der Arbeitgeber schliesslich eine Kün- der Fall ist - zum Schluss, dass die Patientin in ihrer angestammten Tätigkeit in angepasster Weise zu 30\% und in jeder anderen angepassten Tätigkeit zu 100\% arbeitsfähig sei. Somit gewährte der Taggeldversicherer nur noch bis zum 12. Juli 2009 seine Taggeldleistungen.

Im Bemühen um Heilung wurde die Patientin im Mai 2009 zwecks weiterer Abklärung an einen Handspezialisten überwiesen. Dieser diagnostizierte schliesslich ein «Complex Regional Pain Syndrome» (CRPS) Typ I, Stadium III. Dieser Befund wurde dem Taggeldversicherer umgehend zur Kenntnis gebracht, wobei im Arztbericht nur das Krankenbild ausführlich beschrieben wurde, jedoch nicht die Konsequenzen für die Arbeitsfähigkeit. Der Taggeldversicherer nahm diese «neue» Diagnose lediglich zur Kenntnis und reagierte bezüglich seiner Taggeldleistungspflicht nach wie vor unverändert mit dem Hinweis, dass die Patientin in der bereits kommunizierten Weise voll arbeitsfähig wäre.

Diese verzwickte Situation veranlasste die Patientin, sich durch einen Anwalt vertreten zu lassen, der 
auftragsgemäss beim Taggeldversicherer mehrmals versuchte, eine Taggeldleistungspflicht zu erwirken. Ein längeres vergebliches Hin- und Herschreiben zwischen Anwalt und Taggeldversicherer folgte. Der Taggeldversicherer vertröstete den Anwalt stets mit dem Vermerk, dass weitere Abklärungen seitens des Versicherers angeblich noch laufen würden.
Der Patientin blieb gemäss Versicherer nur die Möglichkeit, neue, «qualitativ aussagekräftigere» medizinische Unterlagen zu beschaffen. Diese neuen Forderungen waren, wie sich in der Folge zeigte, nicht einfach umzusetzen. Das Betreuungsumfeld der Patientin hatte mittlerweile eine beträchtliche Grösse erreicht und umfasste als wichtige Instanzen den Hausarzt, den

\section{Ohne die Unterstützung der medizinischen Fachleute bleiben die Rechte der Patienten aber auf der Strecke}

Die zunehmende finanzielle Not der Patientin führte dazu, dass sie im Dezember 2009 einen institutionsunabhängigen Case Manager um Unterstützung bat. Gezielte, hartnäckige Interventionen beim Taggeldversicherer führten schliesslich zu folgenden Erkenntnissen:

- Der Versicherer sah mit dem damaligen Erstellen des vertrauensärztlichen Berichtes alle seine $\mathrm{Ab}$ klärungspflichten als erfüllt an. Weitere Abklärungen waren aus seiner Sicht nicht mehr nötig.

- Aus den ihm zusätzlich vorgelegten «neuen» medizinischen Unterlagen erkannte der Versicherer nach wie vor keine wesentlichen Veränderungen am Gesundheitszustand oder der Arbeitsfähigkeit der Patientin, die seine Taggeldleistungspflicht begründen würden.

\section{Begriffserklärungen und Zusatzinformationen}

\section{Case Management (CM)}

Gemäss «Netzwerk Case Management Schweiz» (www.netzwerk-cm.ch) wird dieser Begriff wie folgt definiert: «CM ist ein spezifisches Verfahren zur koordinierten Bearbeitung komplexer Fragestellungen im Sozial-, Gesundheits- und Versicherungsbereich. In einem systematisch geführten, kooperativen Prozess wird eine auf den individuellen Bedarf abgestimmte Dienstleis tung erbracht bzw. unterstützt, um gemeinsam vereinbarte Ziele und Wirkungen mit hoher Qualität effizient zu erreichen. Weitere Hinweise siehe unter www.iiz.ch (Interinstitutionelle Zusammenarbeit) und www.iiz-plus.ch (Erweiterte Interinstitutionelle Zusammenarbeit). Ziele, involvierte Institutionen und weitere Modalitäten siehe unter www.netzwerk-cm.ch.

\section{FeFi (Früherfassung/Frühintervention)}

Ziel der Früherfassung ist es, möglichst früh jene wegen Krankheit oder Unfall arbeitsunfähigen Personen zu erfassen, die ein Invaliditätsrisiko aufweisen (siehe auch www.iiz.ch/iiz_mamac. aspx). In einer Phase von ca. 6 Monaten soll abgeklärt werden, ob Personen, deren tatsächliche Invalidität noch nicht genau abgeklärt ist, tatsächlich Anspruch auf ordentliche IV-Leistungen haben. Parallel zu dieser Abklärung sollen rasch einsetzende, relativ kostengünstige Massnahmen verhindern, dass Menschen vollständig oder teilweise aus dem Arbeitsprozess herausfallen ([teil]arbeitsunfähig resp. [teil]invalid werden).

Wem wird die Chance geboten, durch Case Management (CM) betreut zu werden?

Nicht jede verunfallte oder erkrankte Person erhält eine Betreuung durch CM. Die Versichere wenden dazu spezielle, intern aufgestellte Kriterien an. Kriterien für eine CM-Betreuung können z. B. unter anderem folgende Aspekte sein: Besteht ein Haftpflichtversicherer (Regressmöglichkeit)? Ist ein UVG-Versicherer zuständig? Ist eine bestimmte Altersgrenze noch nicht überschritten, z.B. 45 Jahre? Besteht ein volles Arbeitspensum? Wird eine bestimmte Einkommensgrenze überschritten? Ist die Person einer der Landessprache einigermassen mächtig? Welche Migrationshintergründe könnten eine Eingliederung behindern? Besteht bereits eine Grundausbildung?
Facharzt, die Psychologin, die Handtherapeutin, die Physiotherapeutin und den bisherigen Anwalt.

Nun galt es, von den Ärzten die medizinischen Unterlagen in der entsprechenden Qualität zu erhalten, die den Taggeldversicherer veranlassen würden, seine Haltung bezüglich seiner Leistungspflicht zu ändern. Das bedeutete, dass sowohl vom Hausarzt als auch vom Facharzt folgende Punkte ausführlich beschrieben werden mussten:

- Krankenbild (Entwicklung, Ist-Zustand, künftige Heilungsmassnahmen und Abschätzung des zeitlichen Ausmasses der Behandlungen. Das Vorlegen von bereits erstellten Unterlagen genügte in diesem Fall nicht).

- Genaue Beschreibung der Auswirkungen des Krankenbildes auf die Arbeitsfähigkeit (vergangenheitsund zukunftsbezogen).

- Kritische, präzise Stellungnahme zu verschiedenen Aussagen aus dem Vertrauensarztbericht. Dies ist unbedingt nötig für eine weitere versicherungsfachtechnische Betreuungsphase - z.B. für die IV-Stelle, für den Taggeldversicherer, für die Arbeitslosenversicherung/RAV-Beratung, für den Pensionskassenversicherer und allenfalls für ein bevorstehendes Gerichtsverfahren.

\section{Schlussfolgerungen}

Obschon die Geschichte dieses Versicherungsfalles bei Drucklegung noch in Bearbeitung ist, zeigt sie in exemplarischer Form auf, wie sich die Ärzte der Patienten in Zukunft mit ihren Bemühungen für den Patienten «neu» orientieren müssen. Unter anderem wird der Schreibaufwand leider immer höher, und die Anforderungen bezüglich der Ausdrucksweise resp. der Ausdrucksqualität steigen zusehends. Das Umschreiben oder Benennen eines Krankenbildes in einfacher Weise genügt im Rahmen der Umsetzungsmodalitäten der 5. IVG-Revision nicht mehr; die zu erwartende 6. IVGRevision verspricht auch keine Erleichterung. Ohne die Unterstützung der medizinischen Fachleute bleiben die Rechte der Patienten aber auf der Strecke.

Die Kurzfassung des geschilderten Falls könnte wie folgt lauten: Der Taggeldversicherer veranlasst auf seine 


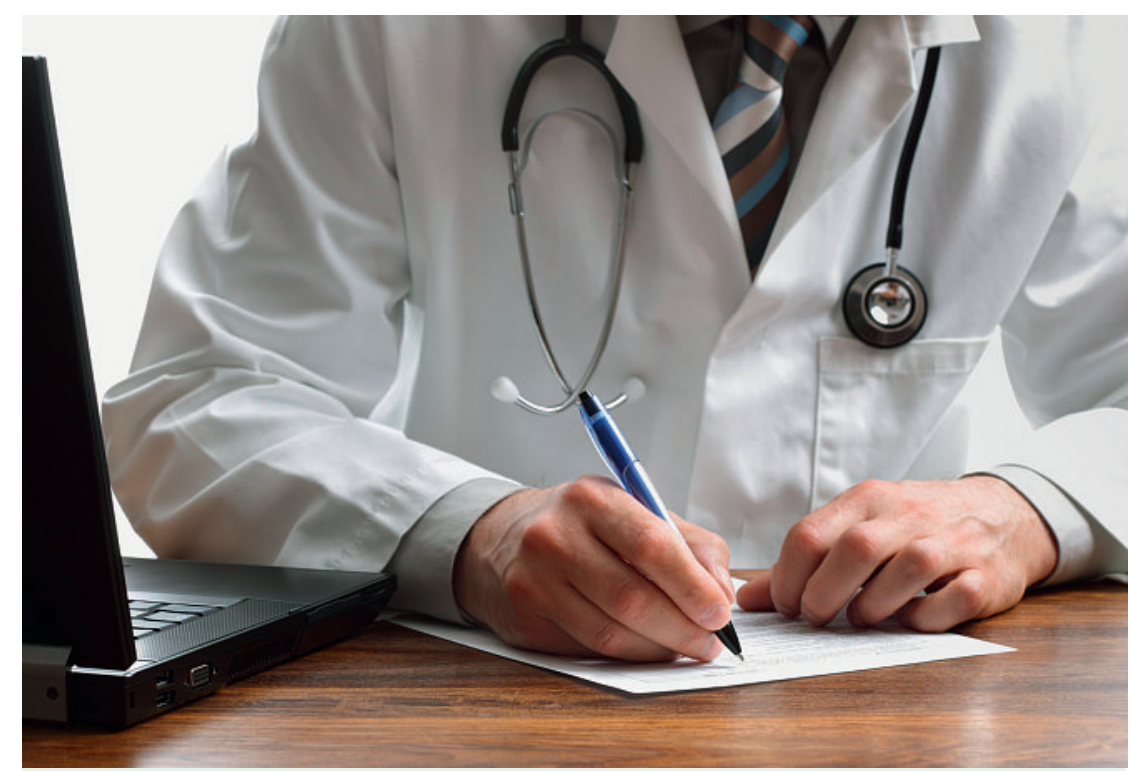

Gibt es in einem Gutachten nicht haltbare Behauptungen und Interpretationen, müssen diese vom Patienten- und Facharzt rasch richtiggestellt werden, damit das Recht des Patienten gewahrt bleibt.
Sicht nicht zutreffendes vertrauensärztliches Gutachten nicht sofort präzis korrigiert resp. die nicht haltbaren «Behauptungen» und «Interpretationen» nicht rasch richtigstellt, so bleibt das Recht des Patienten unberücksichtigt. Ein gerechtes juristisches Urteil für den Patienten ist bei einer solchen ungünstigen Ausgangslage meistens ebenfalls nicht zu erreichen.

Die 5. IVG-Revision (und auch die zu erwartende 6. IVG-Revision) hat bei komplizierteren Heilungsverläufen für die Betroffenen selbst und für das Behandlungsteam nicht unbedingt eine Erleichterung gebracht. Die Versicherungssprache, die Sprache der Mediziner sowie das Denken und Wirken der Gerichte sind drei verschiedene «Welten» - und schliesslich ist da noch die Welt des Patienten. Gerade in Situationen, in denen es darauf ankommt, den Patientenstatus zu stärken, muss der Kommunikation zwischen Patientenarzt, Patient und Versicherer grösste Beachtung geschenkt werden. Bezieht der behandelnde Haus- oder Facharzt des Patienten gegenüber den Versicherern, z. B. aufgrund eines vom Versicherer durch einen gesellschaftseigenen Vertrauensarzt erstelltes Gutachten, nicht sofort sachlich und situationsgerecht Stellung, so drohen dem Patienten daraus erhebliche Nachteile beim späteren Beurteilen seines versicherungstechnischen Leistungsfalles. Die Praxis zeigt leider, dass der administrative Aufwand für das medizinische Patienten-Behandlungsnetz grösser geworden ist. Ein insti-
Kosten in einem Frühstadium des Krankheitsfalles ein medizinisches Arztgutachten. In der täglichen Praxis ist häufig zu beobachten, dass solche Gutachten der Komplexität des Falls nicht gerecht werden und ten-

«Case Management ist ein spezifisches Verfahren zur koordinierten

Bearbeitung komplexer Fragestellungen im Sozial-, Gesundheitsund Versicherungsbereich»

denziell «zugunsten» des Versicherers - und somit «zuungunsten» des Patienten - ausfallen. Der Versicherer hat damit seine Abklärungspflicht erfüllt und kann seine Leistungen «zu Recht» einstellen. Wenn das Ärzteteam um den Patienten herum ein aus seiner tutionsunabhängiger Case Manager als «Übersetzer» zwischen Patientenarzt/-facharzt und Versicherer kann in solch komplexen Situationen für den Patienten hilfreich sein. 